\title{
Longitudinal monitoring of individual infection progression in Drosophila melanogaster
}

\author{
Bryan A. Ramirez-Corona $\ddagger^{1}$, Anna C. Love $\ddagger^{2}$, Srikiran Chandrasekaran ${ }^{3}$, Jennifer A. \\ Prescher $^{2,4-5}$, Zeba Wunderlich ${ }^{1,6,7}$ \\ ${ }^{1}$ Department of Developmental and Cell Biology, University of California, Irvine, Irvine, CA, \\ United States \\ ${ }^{2}$ Department of Chemistry, University of California, Irvine, Irvine, CA, United States \\ ${ }^{3}$ Center for Complex Biological Sciences, University of California, Irvine, CA, USA \\ ${ }^{4}$ Department of Molecular Biology and Biochemistry, University of California, Irvine, Irvine, CA, \\ United States \\ ${ }^{5}$ Department of Pharmaceutical Sciences, University of California, Irvine, Irvine, CA, United \\ States \\ ${ }^{6}$ Department of Biology, Boston University, Boston, MA, United States \\ ${ }^{7}$ Biological Design Center, Boston University, Boston, MA, United States \\ ¥ These authors contributed equally to this work
}

\begin{abstract}
The innate immune system is critical for host survival of infection. Infection models in organisms like Drosophila melanogaster are key for understanding evolution and dynamics of innate immunity. However, current toolsets for fly infection studies are limited in their ability to resolve changes in pathogen load on the hours time-scale, along with stochastic responses to infection in individuals. Here we report a novel bioluminescent imaging strategy enabling non-invasive characterization of pathogen load over time. We demonstrate that photon flux from autobioluminescent reporter bacteria can be used to estimate pathogen count. Escherichia coli expressing the ilux operon were imaged in whole, living flies at relevant concentrations for immune study. Because animal sacrifice was not necessary to estimate pathogen load, stochastic responses to infection were characterized in individuals for the first time. The high temporal resolution of bioluminescence imaging also enabled visualization of the fine dynamics of microbial clearance on the hours time-scale. Overall, this non-invasive imaging strategy provides a simple and scalable platform to observe changes in pathogen load in vivo over time.
\end{abstract}

\section{Introduction}

Bacteria are widespread and can cause severe disease in animals, including humans (Hunter et al., 2010). Hosts mitigate acute illness through deployment of the immune system. Infection progression and outcome is ultimately determined by a combination of genetics, environment, and stochastic events (Carruthers et al., 2020; Duneau et al., 2017). Determining the relative contribution of each of these factors to individual prognosis will enable the identification of genetic markers and early predictors of infection outcome. Such discoveries will contribute to targeted treatment of bacterial infections.

Determination of genetic and stochastic contributions to infection outcome requires a host organism amenable to genetic manipulation and high-throughput experimentation. Drosophila melanogaster fulfills both of these criteria. There are thousands of inbred and sequenced $D$. melanogaster lines, and flies are tractable for high-throughput experimentation in 96-well plates, unlike common mammalian model organisms (Lack, Lange, Tang, Corbett-Detig, \& Pool, 2016). Additionally, Drosophila possess an innate immune system composed of 
signaling pathways that are highly conserved in mammals (Lemaitre \& Hoffmann, 2007). Flies use both the Toll and IMD signaling pathways in their immune response. The Toll pathway was initially discovered in flies and is analogous to the Toll-like receptor signaling pathway found in mammals (Lemaitre \& Hoffmann, 2007). In both flies and mammals, the pathway depends on molecular recognition through pattern recognition receptors (PRRs) to initiate downstream immune response (Moy \& Cherry, 2013). The IMD pathway in flies is orthologous to the TNF receptor family signaling cascade in mammals (Buchon, Silverman, \& Cherry, 2014; Lemaitre \& Hoffmann, 2007). Beyond their use as a platform for discovered conserved immune genes, studying immunity in insects like Drosophila provides insight into how insect vectored diseases spread, and how they may be contained (D. Schneider, 2000).

Variation in infection outcome between $D$. melanogaster lines is in part determined by the genetic backgrounds of the host (Duneau et al., 2017; Hotson \& Schneider, 2015; Lazzaro, Sackton, \& Clark, 2006; Sackton, Lazzaro, \& Clark, 2010). Using genetically distinct D. melanogaster lines, previous work has identified how different loci affect either the ability of an animal to reduce bacterial load or to induce gene expression upon infection (Frochaux et al., 2020; Lazzaro et al., 2006). Less studied both in its mechanism of action and its variation between lines is the fly's ability to endure infection, i.e tolerance. Though studies have shown that the genes involved in infection tolerance somewhat overlap with those involved in resistance, this has yet to be comprehensively determined for different pathogens and across genetically diverse lines (Ayres, Freitag, \& Schneider, 2008; Ayres \& Schneider, 2008; D. S. Schneider \& Ayres, 2008; Troha, Im, Revah, Lazzaro, \& Buchon, 2018).

$D$. melanogaster lines also display variability in infection response within genetically identical individuals. This variation has been indirectly linked to stochastic differences in bacterial growth within the colonized host early in the infection process and variation in the onset of the animal's immune response (Duneau et al., 2017; Ellner, Buchon, Dörr, \& Lazzaro, 2021). The exact source of stochasticity has yet to be directly observed, largely due to a lack of tools capable of providing information on bacterial load noninvasively over time. Characterization of infection progression in flies has typically relied on destructive methods to establish bacterial loads at static time points, e.g. dilution plating. Dilution plating involves sacrificing individuals and quantifying bacterial load using colony counts from serially diluted fly homogenates. While these averaged "snapshot" analyses can provide biological insight into mechanisms of infection progression and clearance, they are unable to capture the stochastic variability that occurs between individuals (Chambers, Jacobson, Khalil, \& Lazzaro, 2019; Kutzer \& Armitage, 2016). Furthermore, fine resolution of early immune dynamics has historically been difficult to determine, owing to low temporal sensitivity of existing methods. New strategies that enable high resolution, non-invasive measurement of pathogen load are necessary for understanding infection dynamics and outcomes of genetically similar and diverse populations.

Historically, a "go-to" method for non-invasive imaging in rodent models is bioluminescence (Love \& Prescher, 2020; Zambito, Chawda, \& Mezzanotte, 2021). Bioluminescence employs luciferase enzymes that oxidize luciferin substrates, producing photons of light (Kaskova, Tsarkova, \& Yampolsky, 2016). These photons can be detected through tissues in whole organisms, enabling sensitive and non-invasive readouts (James \& Gambhir, 2012). Because no external excitation source is necessary, background signals are very low compared to other optical (e.g., fluorescent) readouts (Contag \& Bachmann, 2002). Despite these advantages, bioluminescence has only been sporadically used in $D$. melanogaster (Brandes et al., 1996; Stanewsky, Jamison, Plautz, Kay, \& Hall, 1997; Stempfl et al., 2002). One potential reason for its limited use is that uniform delivery of the luciferin substrate by feeding is difficult, as feeding patterns can vary from fly to fly (Ja et al., 2007). One solution is to use autobioluminescent systems, which produce light without the need for exogenous substrate delivery. There are operons that produce both the luciferase and luciferin, 
allowing transgenic organisms to continuously glow (Kaskova et al., 2016). One popular autobioluminescent system derived from bacteria is the ilux system (Gregor, Gwosch, Sahl, \& Hell, 2018). Engineered from the bacterial lux system, ilux emits blue light $(490 \mathrm{~nm})$ and exhibits enhanced brightness and thermal stability. While autobioluminescent systems have been used for decades to illuminate the spread of various pathogens in vivo (Cronin et al., 2012; Massey et al., 2011; Morrissey, Hill, \& Begley, 2013), they have yet to be applied for studying bacterial clearance in $D$. melanogaster.

Here, we report a novel method employing the ilux system for longitudinally monitoring bacterial load in $D$. melanogaster. By expressing the ilux operon in requisite bacteria and using photon count as a reporter for relative microbial load, we can non-invasively monitor infection progression and clearance over time. With this method, we are able to observe distinct infection dynamics between lines of $D$. melanogaster as well as between genetically identical individuals. This method generated real-time, high-throughput and longitudinal measures of infection in $D$. melanogaster, a feat that has not yet been accomplished in the field.

\section{Results}

Drosophila infection models have historically required animal sacrifice to determine pathogen load, which limits measurements to static time points (Figure 1A). While this method has provided key insights into fly innate immunity and disease progression (Chambers et al., 2019; Chambers Moria, Jacobson, Khalil, Lazzaro Brian, \& Bäumler, 2014; Duneau et al., 2017; Kutzer \& Armitage, 2016; Lazzaro et al., 2006), the fine dynamics of infection progression and tolerance among individuals remains difficult to measure. To address these limitations, we designed a non-invasive imaging strategy to monitor pathogen load over time (Figure 1B). We employed bioluminescent $E$. coli that constitutively expressed the ilux reporter (ilux-Ecoli) as a proof-of-concept platform (Gregor et al., 2018). The load of these autoluminescent bacteria could then be tracked post-injection, with photon flux reporting on pathogen count. Thus, we hypothesized that this method could be used to track differences in immune response between genetically distinct individuals and identify stochastic differences in infection progression within groups of genetically identical individuals. 

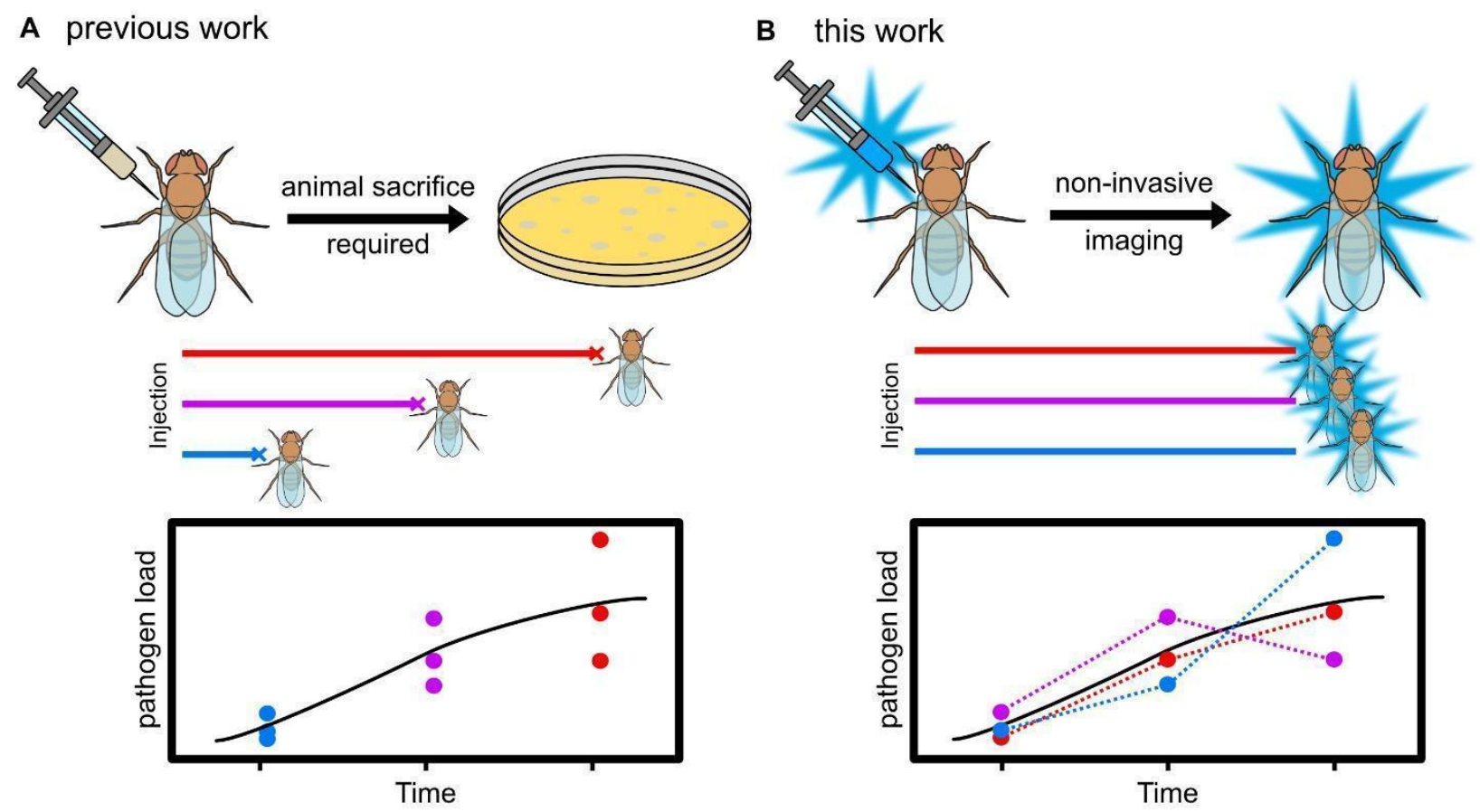

Figure 1. A novel method for non-invasive tracking of pathogen load over time. A) Previous methods for determining pathogen load at static time points require animal sacrifice. Larger cohorts are required for experiments as several flies must be sacrificed at desired intervals to check infection progression and clearance. B) This work presents a novel, non-invasive method to track pathogen load over time using bioluminescence. Thus, all flies can be individually monitored over time, allowing for a more comprehensive view of immune response.

To employ autobioluminscence as a reporter for pathogen count, we first measured how photon flux correlated with bacterial optical density (OD) in liquid culture (Figure S1). To do so, we serially diluted ilux-Ecoli in liquid culture and measured bioluminescent output. Photon counts correlated exponentially with bacterial OD. Since OD can be converted to bacteria colony forming units (CFUs), we can determine relative pathogen load from total flux (Figure 2A).

We then sought to determine how flux correlates with bacterial count when injected into living flies. The ilux system emits blue light, with an emission maximum of $490 \mathrm{~nm}$ (Figure S2). While blue light is difficult to detect in the thicker tissues of mammalian model organisms, the fly cuticle is thin, and thus we hypothesized that the blue emission would be readily detectable in infected flies. Indeed, upon injection and imaging ilux-Ecoli in wild-type, male Oregon-R flies, we were able to reliably detect as few as 1000 CFUs. The thermal noise on the imaging instrument used (IVIS Lumina II) is $\sim 10^{\wedge} 2$ photons/sec. Thus, we could not reliably image <1000 CFU. A positive correlation was observed between CFU and photon flux indicating that flux can report on relative microbial count in vivo (Figure 2B). To determine whether sexually dimorphic pigmentation of the cuticle affected this relationship, we also compared the best fit line of radiance/CFU of male and female flies. We found no differences between sexes (Figure S3) 
Thus, we were confident bioluminescence could be employed to determine pathogen count in both male and female flies. When we compared the relationship between CFU and total flux in liquid culture versus in flies, we found no difference in relationship (Figure S4). This suggests that the fly cuticle does not interfere with photon penetrance, and that we can use standard curves of flies injected with known amounts of bacteria to calculate relative load at a given point in time.

A

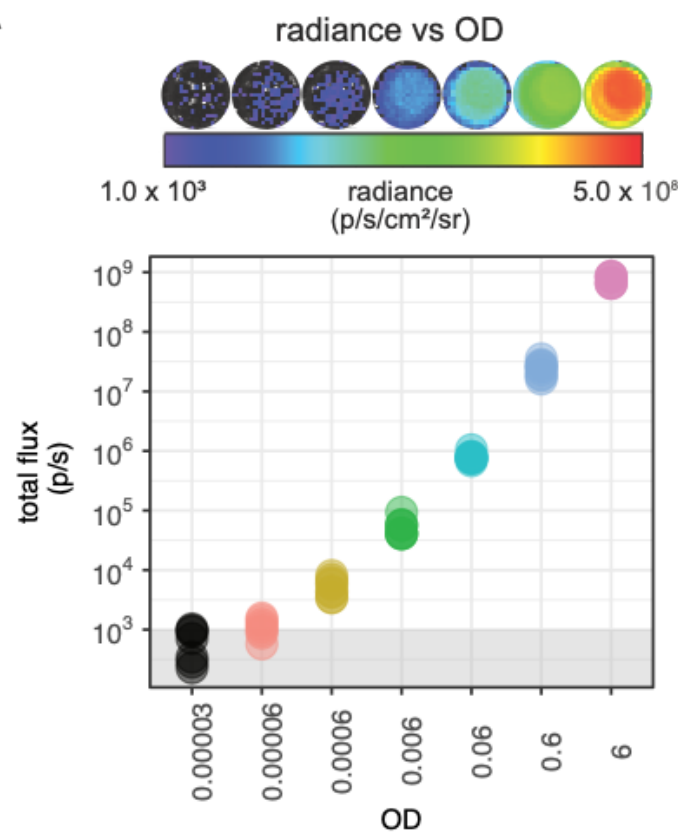

B
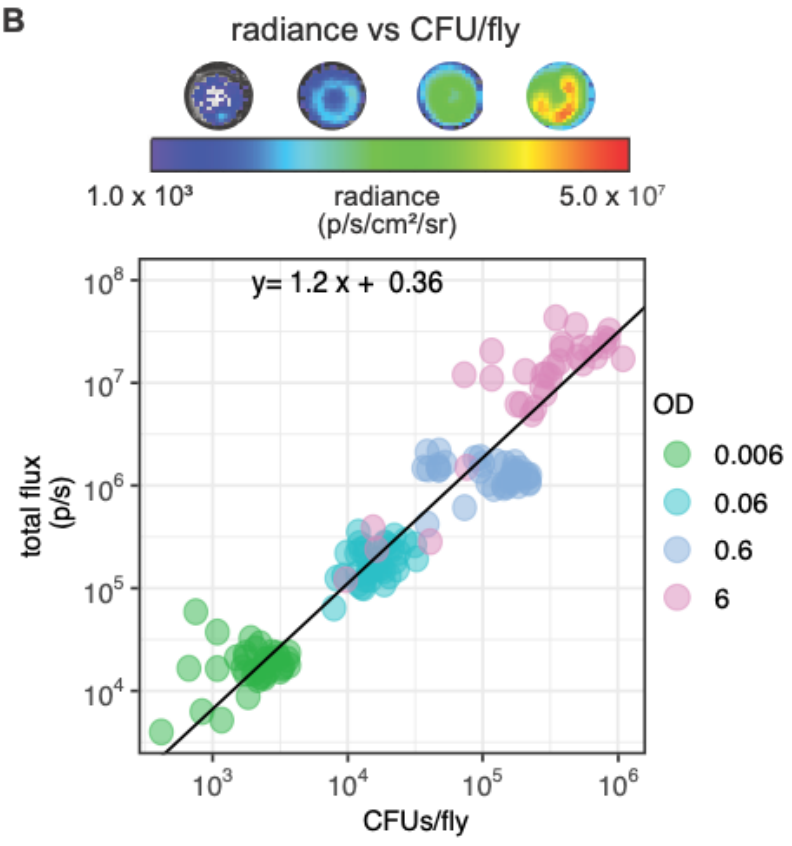

Figure 2. Photon flux is positively correlated to bacterial concentration. A) ilux-Ecoli were serially diluted in liquid cultures and assayed for photon output. Higher concentrations of bacteria correlate with higher photon fluxes. Plot shows twelve measurements per OD (four technical replicates across three biological replicates). Well images are representative of three biological replicates. B) Wild-type flies were injected with different concentrations of ilux-Ecoli and assayed for light emission. A linear correlation was observed between radiance and CFUs injected. Standard major axis regression of the data showed the slope of 1.2 (confidence interval $(\mathrm{Cl}):[1.16,1.29])$. Graph shows data of 36 injected flies. Well images above each graph are representative of images of the injected flies. In both experiments, radiance was summed over the entire well to yield flux using the Living Image software.

To determine whether bioluminescence could be employed for longitudinal tracking of pathogen load over time, we monitored flies for infection progression over several days. Given the duration of the experiment, the flies required housing both compatible with imaging and including food to prevent starvation. To this end, flies were housed in black 96 -well plates by preparing small aliquots of food for each well and placing a glass sheet overtop of the plate (Figure S5). The glass sheet was secured with black electrical tape to mitigate aberrant photon scattering. With the housing in hand, we infected 48 flies with increasing concentrations of ilux-Ecoli and transferred them to individual wells in the prepared housing. The flies were then imaged for four days, and photon fluxes recorded (Figure 3A-B). Since the flies were freely moving during the 3-5-minute image acquisition, they created a donut shaped signal by traveling around the sides of the well (Figure 3A). We can account for fly activity in quantification by summing the photon count of the entire well. 
Since wild-type flies mount a robust immune response against $E$. coli infection, none of the flies died during the course of the experiment. For lowest doses of bacteria administered, photon flux values indicated that the infection had been cleared to levels $<1000 \mathrm{CFU}$, i.e., below the detection limit. At higher doses, detectable amounts of bacteria remained on day 4 . Further monitoring would distinguish whether flies exposed to higher initial doses of $E$. coli maintain a systemic, but non-lethal, infection (Chambers et al., 2019; Chambers Moria et al., 2014) or whether more time is needed to clear the infection. To ensure the decreasing photon counts as a function of time were due to infection clearance rather than loss of the ilux plasmid, we performed the same time course and performed bioluminescence imaging of plated fly homogenate for each day (Figure S6, S7). We observed minimal plasmid loss. Clearance patterns varied among flies receiving the same dose of pathogen (Figure 3C). For example, when looking at the trajectories from the two lowest doses, there are individual flies that experience resurgent infections, which are unobservable with dilution plating assays. Some flies receiving the highest dose show markedly faster clearance dynamics than others. Our longitudinal measurements allow us to determine that this is true variation in the infection response, as opposed to differences in initial dose. Together, these results highlight how bacterial bioluminescence can be used to image stochastic responses to infection.

A
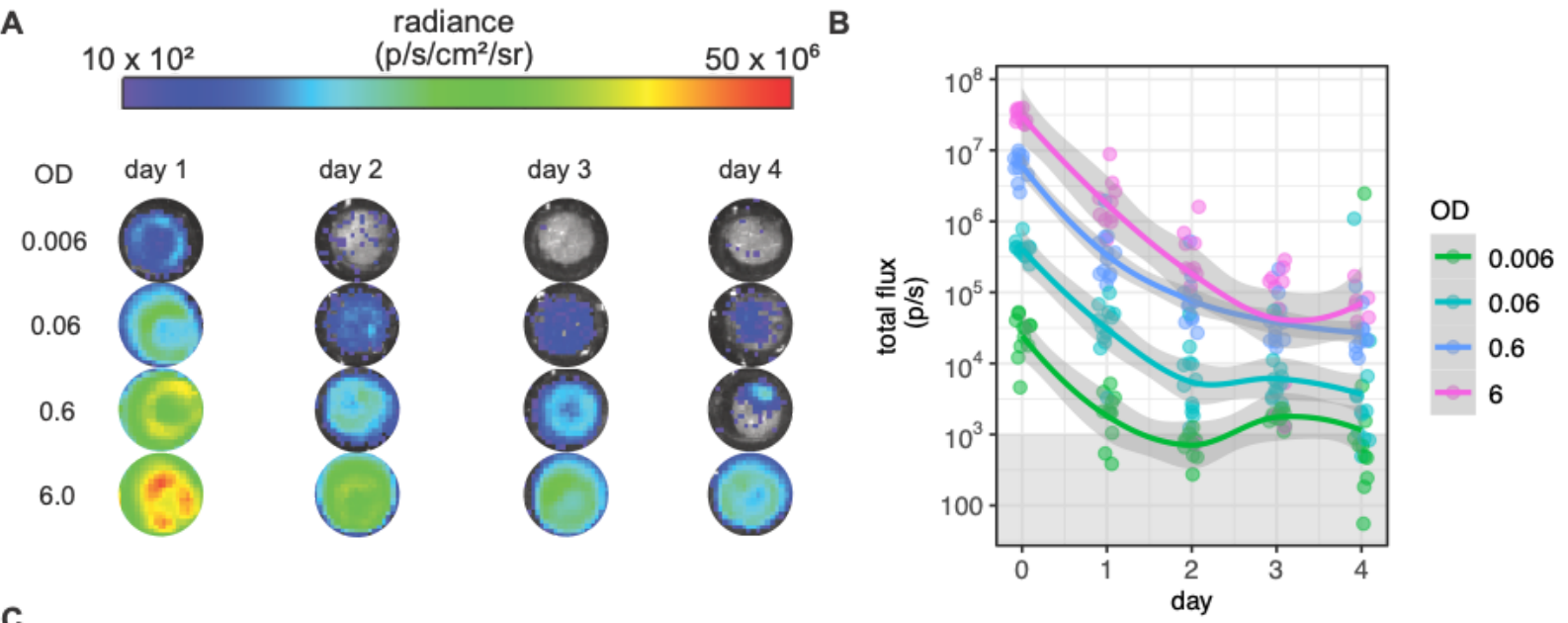

C
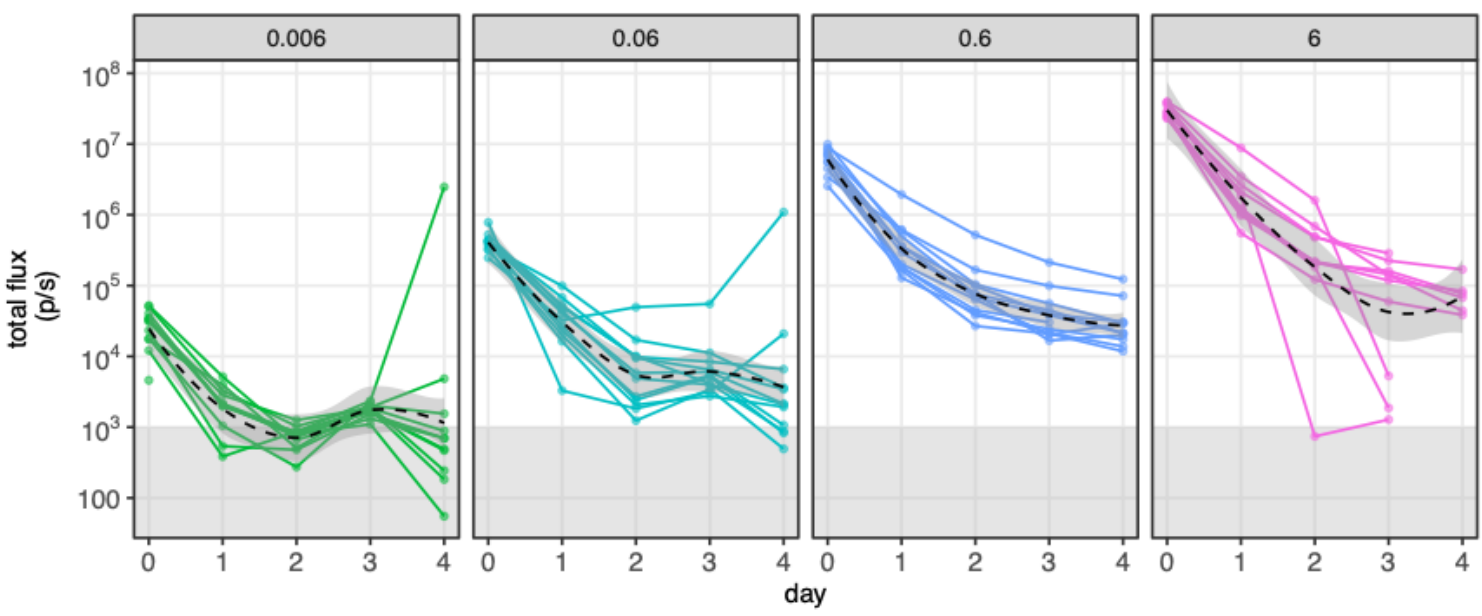

Figure 3. Bioluminescence can be used to track changes in pathogen load over time. A) Representative well images for different concentrations of bacteria injected in wild-type flies over four days. Higher initial concentrations were cleared to low, but detectable concentrations. Low 
initial concentrations were cleared below the limit of detection for the imaging instrument. Well images are representative of 48 injected individuals. B) Average clearance patterns for different concentrations of ilux-Ecoli injected in individual flies over time. Photon counts were summed over the entire well where flies resided. Grey box shows the limit of detection of the imaging instrument. Solid colored line represents the average of the cohort. Grey bar represents the standard deviation over replicates, dots represent one individual. Graph shows data of 48 injected flies. C) Individuals display varied routes toward infection clearance, suggesting stochasticity plays a role in infection dynamics. Black dotted line represents the average, and the grey band represents the standard deviation. Grey box shows the limit of detection of the imaging instrument. Solid lines represent routes individuals took toward infection clearance. Data shown were taken from 48 infected individuals.

Historically, stochasticity of immune response in the first 12 hours of infection has been difficult to observe due to the need for fly sacrifice to obtain pathogen load information. We hypothesized our bioluminescent imaging strategy would be useful for studying differences in pathogen clearance during these critical first hours of infection. For example, we can measure when the immune system is activated, which may vary between individuals, genotypes or in response to different pathogens. To test this capability, we used two fly lines, Oregon-R wild-type flies, and immunodeficient imd ${ }^{10191}$ flies (Pham, Dionne, Shirasu-Hiza, \& Schneider, 2007). Wild-type flies are resilient to $E$. coli infection and clear the Gram-negative microbes easily, as shown in Figure 3. Imd ${ }^{10191}$ flies, by contrast, more easily succumb to infection. These organisms bear a frameshift mutation in the IMD protein, effectively eliminating the immune response to Gram-negative bacteria. Therefore, we anticipated imd ${ }^{10191}$ flies would have different pathogen loads compared to wild-type files. Indeed, upon injection of a large quantity $(1,000,000 \mathrm{CFU})$ of ilux-Ecoli, imd ${ }^{10191}$ flies sustained high pathogen levels over time, while Oregon-R flies steadily cleared the infection as evidenced by reduced emission levels (Figure 4B). When handling highly concentrated pathogens for injection, variance in initial dose can occur. In this experiment, the imd ${ }^{10191}$ flies received a slightly lower initial dose of bacteria than the wild-type flies. However, by the two-hour time point, the imd ${ }^{10191}$ flies carried a higher load than the wild-type flies. Although we aim to deliver consistent initial doses, this result highlights a feature of this method: we can censor individual animals that receive aberrant initial doses. This quality control step is not possible with dilution plating-based methods.

Beyond quality control, we can actually use variation in initial dose to answer biological questions. For example, it has been shown that within a genotype variation in initial dose contributes to the differences in bacterial load of chronic infections observed, though this study relies on group averages and is unable to assess the impact of initial infection load (Chambers et al., 2019). To address this question, we plotted the infection dynamics of individual flies (Figure 4C). In the imd $^{10191}$ line, one individual received a low inoculation of bacteria. To determine if the initial load of infection contributed to the differences in bacterial load at the end of the time course, we divided samples in each genotype as having received an initial dose above the mean (high dose) or below the mean (low dose) and tested for differences in the mean of the final bacterial loads. We found the initial dose of pathogen does not correlate with differences in the final load (Oregon-R high vs low: $p=0.18$, imd ${ }^{10191}$ high vs low: $p=0.78$, Welch two sample t-test, Figure S8). The bioluminescent method thus reports on initial inoculation differences and can provide insight into alternative hypotheses for variance in infection dynamics among genetically identical populations. 
A
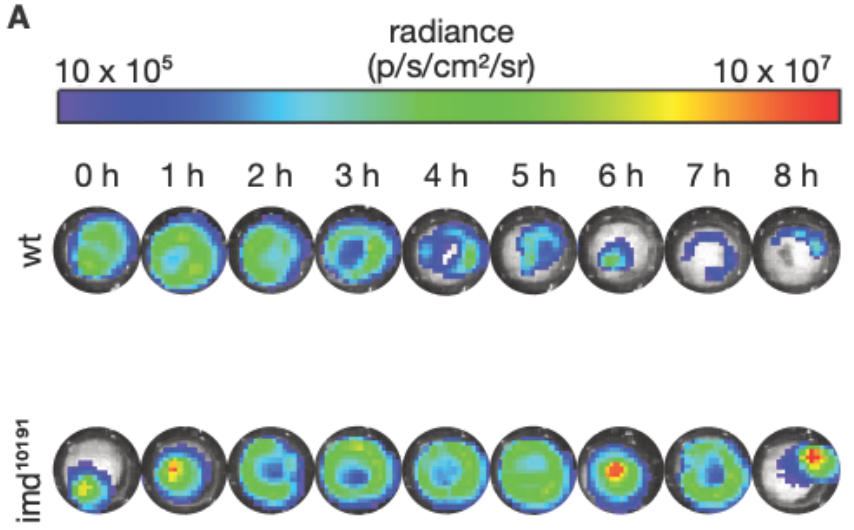

C

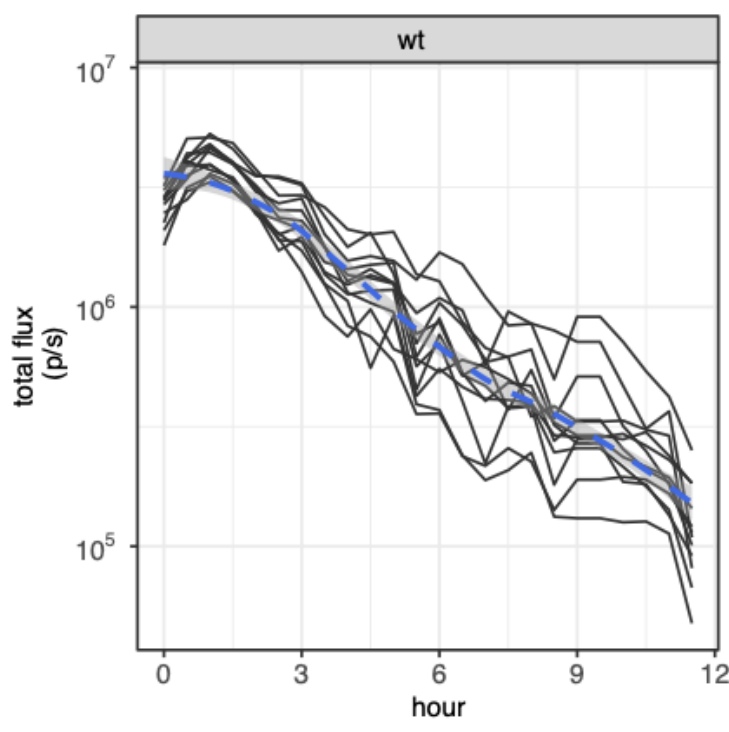

B

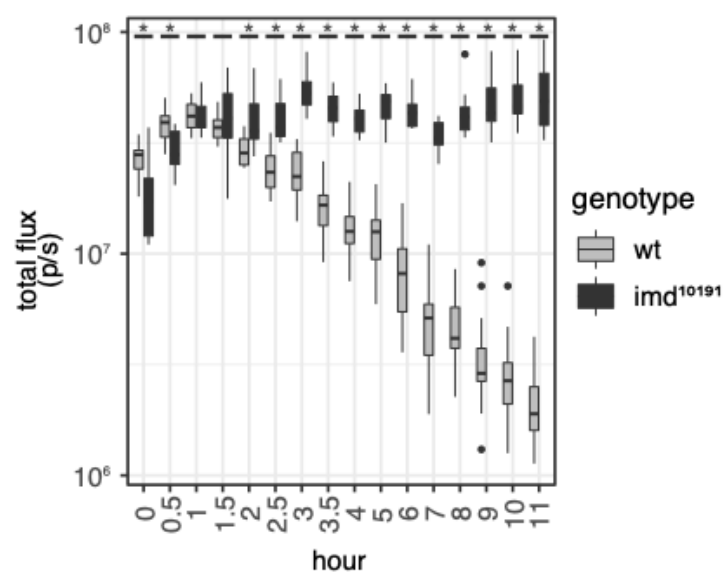

Figure 4: Longitudinal tracking of individual flies allows for deconvolution of community dynamics A) Representative images of radiance measurements for wt and imd ${ }^{10191}$ injected with $.034 \mu \mathrm{l}$ of $\mathrm{OD}=6(1,000,000 \mathrm{CFU})$ ilux-Ecoli. Images show the first 8 hours, after which most imd $^{10191}$ flies perished. Images are representative of 24 injected individuals. B) Comparison of population level integrated total flux. Timepoints showing difference in mean between the two lines are demarked with an asterisk. Immunodeficient lines received a lower dose of infection than wt flies, but within an order of magnitude difference. This significance was lost by hour 1 , with imd ${ }^{10191}$ bacterial load surpassing that of $w t$ by hour 2. Data comprise 12 injected individuals. C) Comparison of wt and imd ${ }^{10191}$ individual tracks. Individual variation of immune response and pathogen clearance was observed in living flies (solid lines). Deaths are marked by red triangles, and the lines end. The blue dotted line shows the average of the cohort, while dark grey lines show the individual paths toward clearance or death. Death histogram shows the effective pathogen load upon death as a function of total integrated flux. The red line on the imd $^{10191}$ graph also demarcates the average of these values. The light grey line indicates a fly that received a lower-than-average initial dose. Thus, these data were filtered out in the subsequent analysis. Data shown comprise 12 injected individuals. 
In the experiment above, the high initial dose of bacteria killed the immune-deficient flies in a small-time window, which made it difficult to assess potential drivers of death. We posited that a lower dose of bacteria would kill immunodeficient flies more slowly and with greater variation in the time to death. To test this hypothesis, we injected wild-type and imd ${ }^{10191}$ flies with 10,000 CFUs of ilux-Ecoli. We then imaged flies every hour for 2 days post-infection. While both strains of flies were injected with the same concentration of bacteria, within the first hour, pathogen load differed between the two populations (Figure 5A-B). Over time, the wild-type flies cleared the infection to low pathogen load and survived to the end of the experiment. Conversely, the imd $^{10191}$ flies showed a gradual increase in pathogen load over time, with all flies succumbing to the infection by the end of the experiment. While initial loads varied between individuals, inoculation load did not correlate with final bacterial load upon death for imd ${ }^{10191}$ individuals, or upon end of experiment for wild type individuals (S8, S9). This suggests variance in initial load does not fully explain variation in bacterial load over time. In line with our hypotheses, we did observe substantial variance in time to death among the imd ${ }^{10191}$ individuals (Figure 5C).

A

radiance

$10 \times 10^{2}$ $\left(\mathrm{p} / \mathrm{s} / \mathrm{cm}^{2} / \mathrm{sr}\right)$

$50 \times 10^{5}$

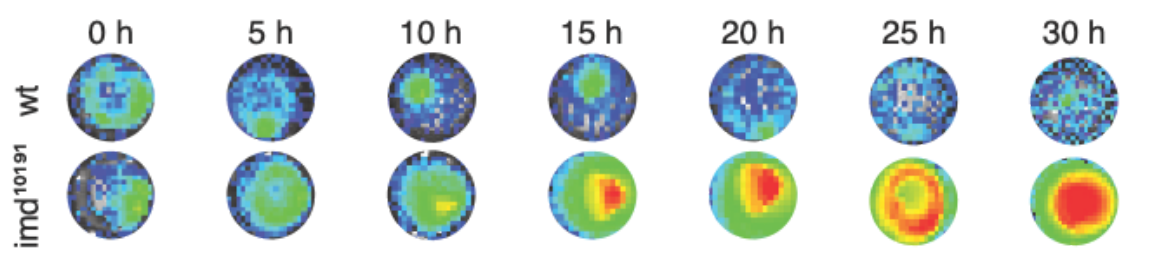

B

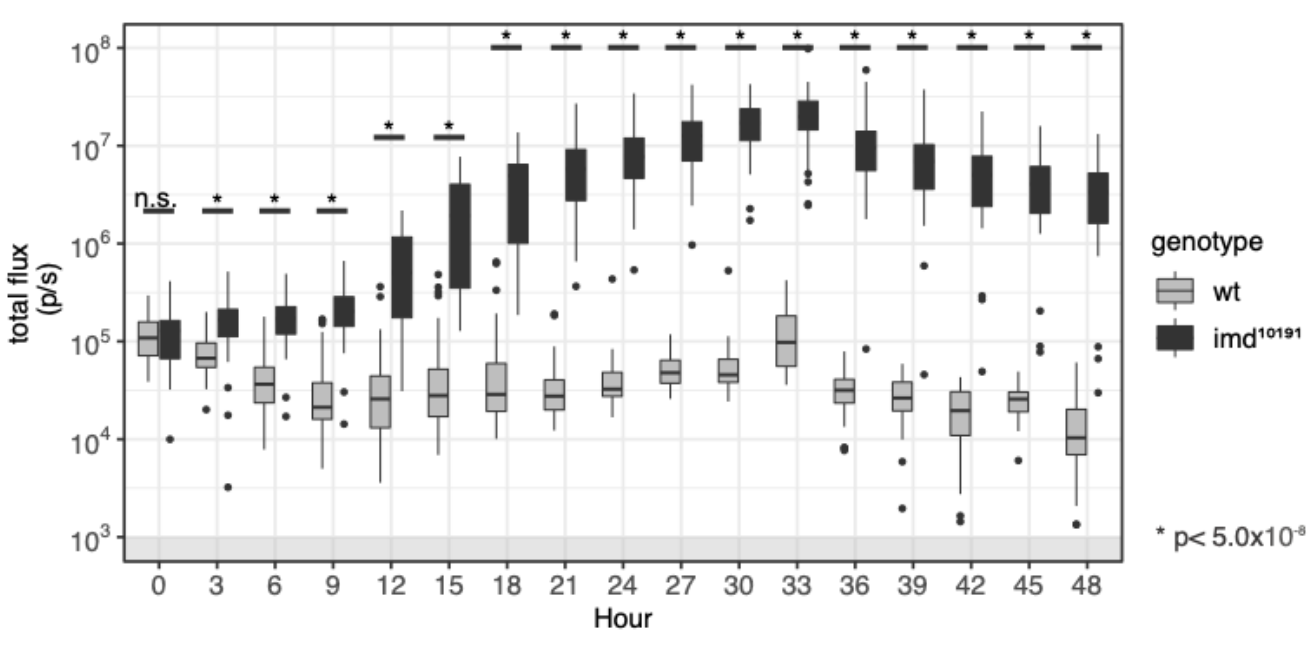

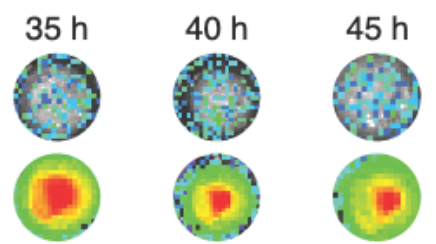

C

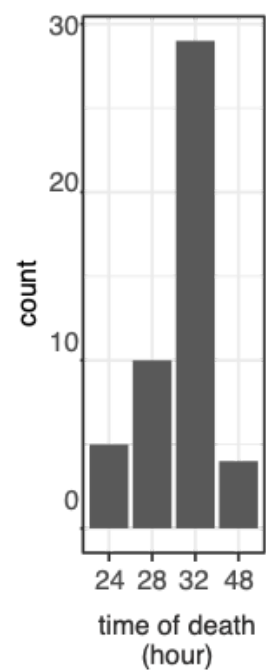

Figure 5: Individual infection tracking of immune-deficient flies allows for deconvolution of infection variation. Both wt and imd ${ }^{10191}$ flies were injected with $0.034 \mu \mathrm{L}$ of OD=0.06 $(10,000$ CFU) ilux-Ecoli ( $\mathrm{n}=48$ for each genotype) A) Representative images of radiance measurements for wt and imd ${ }^{10191}$ flies injected with ilux-Ecoli. Images are representative for 48 individuals and are shown in 5-hour intervals for the first 45 hours of infection. B) Summary of integrated total flux values for the wt and imd ${ }^{10191}$ in 5-hour intervals for the first 45 hours. Timepoints showing difference in mean between the two lines are demarked with an asterisk. By hour 5 , both lines 
show differences in the ability to fight off infection with wild-type flies observed clearing the infection and imd ${ }^{10191}$ flies much higher bacterial loads. C) Histogram displaying time of death statistics for imd ${ }^{10191}$ flies. The majority of imd ${ }^{10191}$ flies died at hour 32 , when integrated total flux reached its highest peak. Death data were compiled from 48 imd $^{10191}$ individuals.

To further explore the variation in the infection progression in immunodeficient flies, we plotted their individual dynamics (Figure 6A). To identify groups of individuals showing distinct infection profiles, samples were hierarchically clustered using Euclidean dissimilarity (Figure 6B, Figure S10, (Montero \& Vilar, 2014)). This clustering requires that each individual have a measurement from all time points; therefore, we included bacterial load data post-mortem for flies that died prior to the end of the experiment. We separated the trajectories into four clusters, with the majority of flies falling into cluster 2 (blue, $n=29$ ) or cluster 3 (yellow, $n=13$ ), and clusters 1 and 4 having three flies each (magenta and green, respectively). Clusters 2 and 3 appear to separate based on the lag time to unchecked bacterial growth, with cluster 2 having a lag time of 5-10 hours and cluster 3 having a lag time of 10-15 hours after infection. Clusters 1 and 4 appear to cluster primarily based on receiving a below-average initial dose (cluster 4 ) or on bacterial dynamics post-mortem (cluster 1). No cluster appeared to correlate with time of death (Welch two sample t-test on every cluster combination, Figure S11). While all samples ultimately succumbed to the infection, these distinct dynamics would have been missed using previously established methods.

A

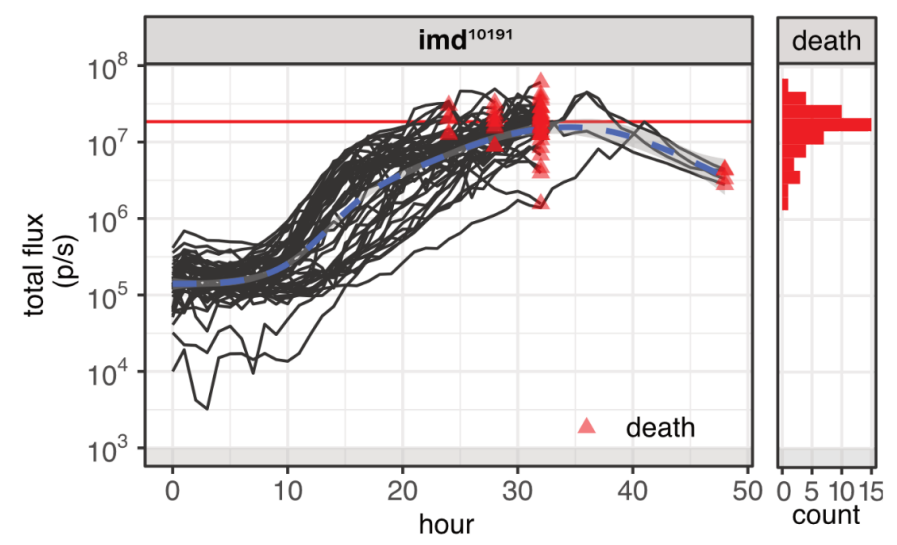

C

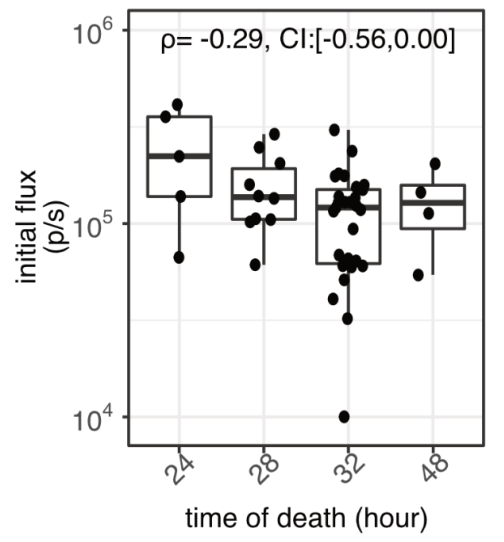

B

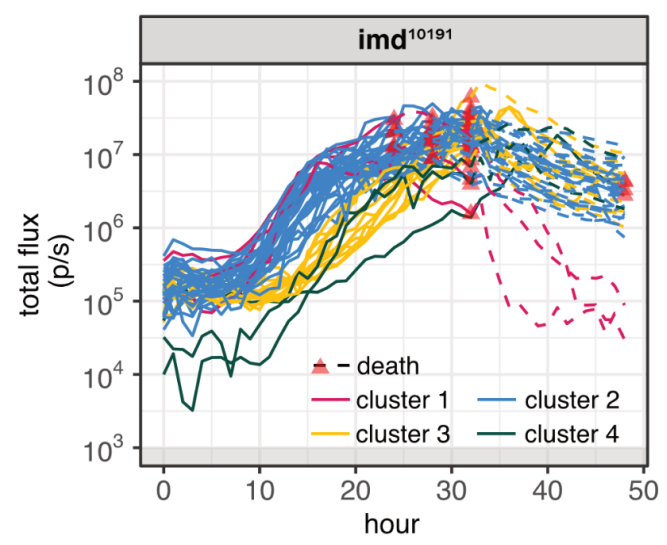

E

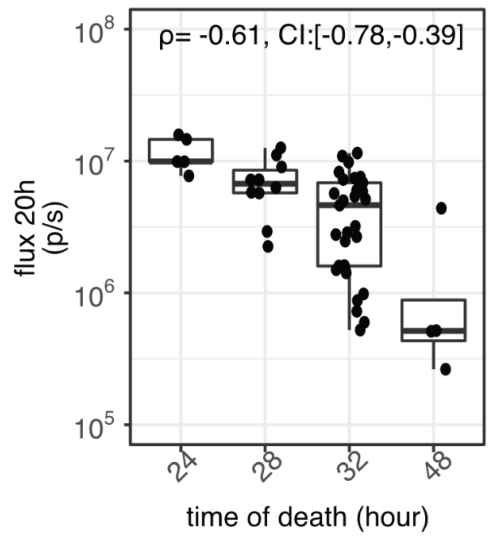

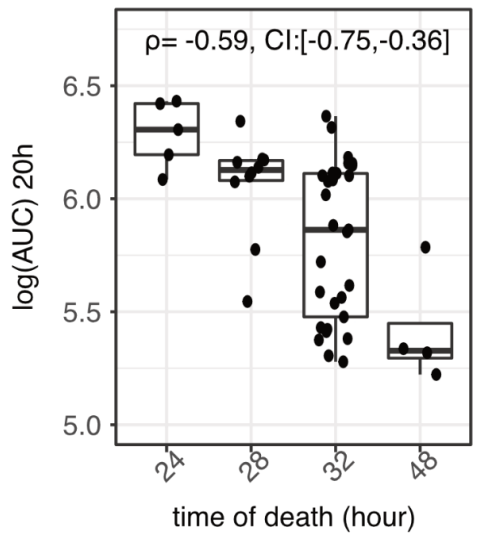


Figure 6: Individual infection tracking of oregon-R flies shows two distinct pathways towards bacterial clearance. A) Individual tracks of infection in live imd ${ }^{10191}$ flies (black lines, $\mathrm{n}=48$ ); deaths are marked as a red triangle and the end of the line. All flies died by hour 48 and the mean radiance upon death was $18.6 \times 10^{6}$ (solid red line). Threshold of accurate detection demarcated with a grey box. Histogram shows the distribution of integrated photon flux (serving as a proxy for pathogen count) of imd ${ }^{10191}$ flies upon death. B) Individual tracks of infection colored by clusters. Clusters were assigned via hierarchical clustering using Euclidean dissimilarity. Four distinct groups were assigned with cluster 2 and 3 containing the majority of samples (cluster $2=29$, cluster $3=13$ ) and clusters 1 and 4 containing 3 samples each. Data are for 48 imd $^{10191}$ individuals. C) Spearman rank correlation $(\rho)$ and the $95 \%$ bootstrapped confidence interval $(\mathrm{Cl})$ between initial flux and time of death for imd ${ }^{10191}$ flies. D) Spearman rank correlation and $\mathrm{Cl}$ between total flux at hour 20 and time of death. E) Spearman rank correlation and $\mathrm{Cl}$ between the log transformed area under the curve (AUC) up until hour 20 and the time of death. The curve here refers to the bacterial load (CFU) vs time curve. In all cases $\mathrm{Cl}$ of Spearman correlation coefficients were computed by bootstrapping 10,000 synthetic datasets and computing the correlations on these datasets. 2.5 and 97.5 percentile values of the sampled correlations are reported.

We further investigated potential sources of the variability observed in time to death of the imd ${ }^{10191}$ flies. Several possibilities exist to explain these differences in dynamics, including variation in the injury upon infection, variation in the initial pathogen load, or differences in the physiological state of the fly. To examine if this variance in time to death can be explained by differences in the initial load of infection, we performed Spearman rank correlations between time of death and the initial load. We also measured the correlations between time of death and load at $20 \mathrm{~h}$ post injection and the area under curve (AUC) of pathogen load at $20 \mathrm{~h}$. We found initial load was negatively correlated with time of death $(\rho=-0.30,95 \%$ bootstrapped confidence interval $=(-0.56,0.00)$ ) (Figure $6 \mathrm{C})$. Intuitively, this aligns with expectations: flies receiving higher inoculations will die more quickly. The strongest correlation was found between time to death and observed load at $20 \mathrm{~h}$ post injection ( $\rho=-0.61,95 \%$ bootstrapped confidence interval $=(-0.78,-0.39))$ (Figure 6D). A similarly strong correlation was observed between time to death and area under the flux curve at $20 \mathrm{~h}$ post injection $(\rho=-0.59,95 \%$ bootstrapped credible interval $=(-0.75,-0.36)$ ) (Figure 6E). These correlations suggest that a threshold may exist where host colonization can no longer be contained by the immune system, and death becomes inevitable. This highlights how factors beyond initial load contribute to the time of death observed in individual flies, and the importance of stochasticity in infection outcome. Indeed, the variable paths flies take toward a potential "point-of-no-return" in bacterial colonization can now be investigated in fine detail using the temporal resolution afforded by our bioluminescent method. Ongoing work involving integration of additional luminescent reporters to label immune system components would enable even more thorough investigation into how different variables can contribute to infection outcome.

Unlike the immunodeficient line, all of the wild-type flies survived well past two days, with the overall trend of clearing the infection (Figure 7A). Despite the average drop in pathogen load, we did observe large amounts of variance, particularly between hours 10 and 20 of infection. Hierarchical clustering sorted the individual traces into groups that corresponded to early pathogen load (Figure S12). In studying this data, we noticed that some individuals experienced a resurgence in infection, and these individuals fell into more than one cluster. Therefore, we highlighted profiles based on the presence of a secondary distinct increase of pathogen load after the initial infection (magenta, $n=10$ ) rather than a sustained gradual decrease (blue, $n=38$, Figure 7B). Again, we found the initial dose of infection did not correlate with the presence of a secondary peak (Oregon-R C1 vs C2: $p=0.41$, Welch two sample t-test, 
Figure S13). Despite the presence of an increase in pathogen load in some flies, the population ultimately converges towards clearing the infection. We expect that this method's ability to report fine differences in pathogen load over time will enable future research to uncover the origins of such stochasticity and better characterize the paths hosts may take to clear an infection.

A

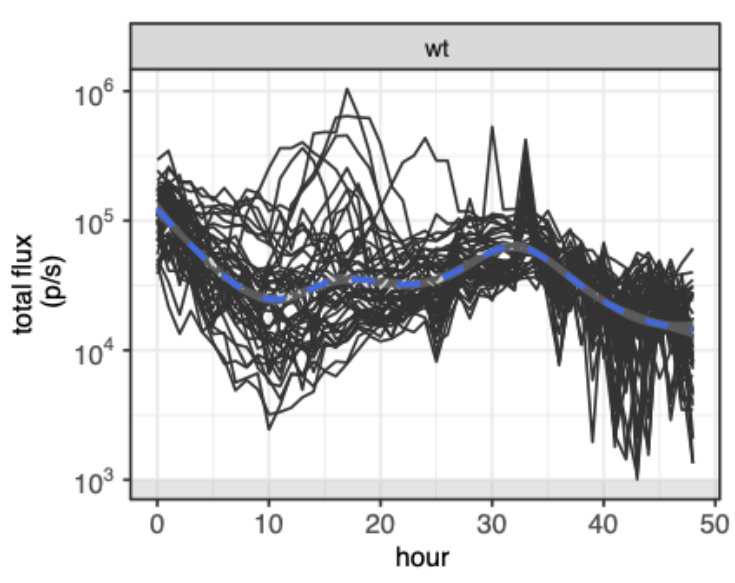

B

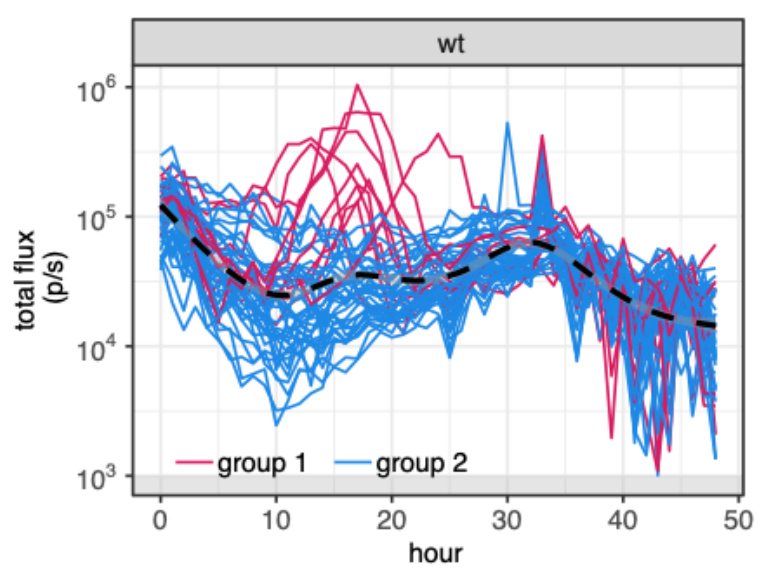

Figure 7: Individual infection tracking of wt flies shows two distinct pathways towards bacterial clearance. A) Individual tracks of infection in live flies (black lines). No flies died during this time course. Threshold of accurate detection demarcated with grey box. B) Individual tracks of infection grouped by the presence of a secondary peak during the infection process. While all flies showed a decrease in bacterial load by the 48 hour mark, a subset of flies $(n=10)$ showed an increase in bacterial load between 10 - 25 hours (magenta lines). Dashed lines represent the mean trajectory for the genotype.

\section{Discussion}

Here we show that employing an autobioluminescent bacterial reporter enables simple, non-invasive pathogen load determination in Drosophila melanogaster. We show that total flux can report on changes in bacterial load over time. The non-invasive feature of this method fundamentally changes our view into infection dynamics by enabling longitudinal tracking of infections in individual animals. Traditional dilution plating approaches only allow for the measurement of an average infection trajectory in a population of genetically identical individuals, while longitudinal measurements allow us to reveal individual variation in dynamics and features such as resurgent infections. Further, these traces can be used to test hypotheses about what features (e.g. initial dose, time to immune system engagement, or colonization progress) drive the ultimate outcome of infection. The simple housing requirements and rapid acquisition times allow for the efficient measurement of large numbers of animals or genotypes at fine time resolution. Because each animal is repeatedly sampled throughout the experiment, the longitudinal measurements effectively reduce the sample size needed to identify differences in distinct genotypes of flies. The decrease in sample size, coupled with the increase in throughput, will make previously laborious genetic screens to identify new components of the immune response more accessible. 
The flexibility and simplicity of this method should enable its use in a wide range of settings. For example, the ilux cassette can be manipulated via molecular cloning, allowing expression in a wide variety of pathogens. The fly housing requires only simple components found in most laboratories. We also examined the feasibility of using a plate reader instead of an IVIS imager for luminescence measurements (Figure S15). Using flies injected with bacterial doses spanning 6 orders of magnitude, we found that while the IVIS has better sensitivity in detecting low pathogen loads, the plate reader performed comparably to the IVIS at higher doses. This indicates that the plate reader may be useful for examining infections leading to binary outcomes such as death.

The longitudinal measurements and temporal resolution achieved using bioluminescence enables novel observations of infection dynamics. For example, although we found wild type flies cleared ilux-Ecoli in all experiments, low initial doses prompted a resurgence in infection among certain individuals (Figure 7). Using previous dilution plating methods, resurgence cannot be definitely identified. It would appear to be variation between sacrificed individuals at discrete time points. Using our bioluminescent method, resurgence can be easily visualized as an increase in total flux observed in certain individuals of a population and future studies may investigate the causes and predictors of a resurgent infection.

New insights were also gleaned from experiments with immunodeficient flies. We found a moderate correlation between the initial load and time to death for immunodeficient flies, and a stronger correlation between time to death and bacterial load at $20 \mathrm{~h}$ post injection for individuals receiving a low initial dose of ilux-Ecoli (Figure 6D). This correlation suggests that flies may have a bacteria "threshold" where colonization proceeds unchecked, and death becomes inevitable (Duneau et al., 2017). Taken together, it seems both variation in initial load and infection progression combine to determine the ultime time of death. Thus, bacterial loads at earlier timepoints may be able to predict whether survival or death may be expected.

In summary, non-invasive tracking of pathogen load in Drosophila melanogaster over time offers many advantages when compared to traditional methods. Dilution plating requires animal sacrifice to determine average pathogen load at static time points, limiting investigations into individual infection dynamics. Fine differences in pathogen load, infection progression, and immune activation are unable to be determined. The bioluminescent method presented herein offers a facile approach to non-invasively monitor pathogen load on the individual level. Because images may be acquired on the minute-time scale, bioluminescence enables infection progression to be monitored with exceptional resolution. Using this method, we can begin to quantify the contributing factors that result in stochasticity, resurgence, and ultimate infection outcome.

\section{Acknowledgments:}

We would like to thank Dr. Larry Marsh and Dr. Neal Silverman for sharing their fly lines to make this work possible and $\mathrm{Zi}$ Yao for his assistance with the pilot experiments. This work was supported by NSF/BIO/MCB grant 1953324 to ZW, a Convergence Accelerator Team award from NSF-Simons Center for Multiscale Cell Fate Research to JP and ZW, and a seed grant from the UCI Infectious Disease Science Initiative to JP and ZW.

\section{Materials and Methods}




\section{Preparing ilux E. coli}

An E. coli strain harboring a plasmid with the ilux operon (ilux pGEX(-)) was obtained from Addgene (plasmid \# 107897, deposited by Stefan Hell) and streaked on an LB agar plate with ampicillin $(100 \mu \mathrm{g} / \mathrm{mL})$ to afford single colonies. A single colony was picked and grown in $5 \mathrm{~mL}$ LB broth containing ampicillin (100 $\mu \mathrm{g} / \mathrm{mL}$, LB-AMP). The culture was miniprepped according to the manufacturer's instructions (kit purchased from Zymo Research). The concentration of the plasmid was determined using a NanoDrop 3000 (Thermo Fisher). Plasmid (10 ng) was transformed into chemically competent TOP10 E. coli $(20 \mu \mathrm{L})$. The transformant was recovered with SOC $(50 \mu \mathrm{L})$ for 30 mins at $37^{\circ} \mathrm{C}$ and $25 \mu \mathrm{L}$ plated on an agar plate containing ampicillin. A single colony was picked and expanded in LB-AMP, and a glycerol stock was made for long term storage at $-80^{\circ} \mathrm{C}(500 \mu \mathrm{L}$ culture with $500 \mu \mathrm{L} 50 \% \mathrm{v} / \mathrm{v}$ glycerol $)$. This glycerol stock is referred to as ilux-Ecoli.

\section{Drosophila lines and rearing}

Oregon R and imd-10191 were used for this study (Pham et al., 2007). Both lines were reared on standard cornmeal media at $20^{\circ} \mathrm{C}$ (Brent and Oster 1974). Four-day old male and female flies were collected for injections to ensure full replacement of the larval fat body by the adult fat body (Johnson \& Butterworth, 1985).

\section{Drosophila infection induction}

Prior to infection, ilux-Ecoli was cultured in liquid LB-AMP on a shaker at $37^{\circ} \mathrm{C}$ for 8 hours. Bacteria were then pelleted using a table-top micro-centrifuge at $5000 \mathrm{rpm} / \mathrm{g}$ and resuspended in $200 \mu \mathrm{l}$ of $1 \mathrm{X}$ phosphate buffered saline. Optical density was then measured using a NanoDrop 2000 (Thermo Fisher). Injection solutions were prepared at the appropriate OD by dilution in additional PBS. Flies were injected with $34 \mathrm{~nL}$ of bacterial solution using Narishige IM 300 Microinjector along the scutescutullar suture and immediately placed into black 96 -well plates (Grenier Bio One). For time course experiments, 96-well plates for imaging were prepared by punching out circles of standard cornmeal media and placing these at the bottom of wells before placing flies into the plate. A 4-inch by 6 -inch glass cover was placed on the plate during the duration of the time course to prevent individual escape. For single time point measurements, flies were placed in 96-well plates lacking food.

\section{Dilution plating: ilux-Ecoli}

To determine the concentration of ilux-Ecoli at per OD measurement, ilux-Ecoli was grown in 10 $\mathrm{mL}$ LB-AMP on a shaker at $37^{\circ} \mathrm{C}$ for 6-7 hours while the bacteria was still in exponential growth phase. Bacteria was then pelleted down and resuspended in $200 \mathrm{uL} 1 \mathrm{X}$ phosphate buffered saline and OD of the solution was determined using a NanoDrop 2000 (Thermo Fisher). A stock solution of OD 1 was prepared and serially diluted using $1 \times$ PBS by 6 million fold. CFUs where then quantified in two ways. The first manner was using $5 \mu$ of solution for each dilution which was spot plated in triplicate on LB plates supplemented with $100 \mu \mathrm{g} / \mathrm{ml}$ ampicillin. The second manner was using $90 \mu \mathrm{l}$ of solution which was plated on LB plates supplemented with $100 \mu \mathrm{g} / \mathrm{ml}$ ampicillin. Colonies were then counted for each dilution step to determine the concentration of CFUs at OD1 for both methods (Supplementary Figure 1). To determine the relationship between ilux-Ecoli concentration and total flux, a solution of bacteria was prepared as described above, $90 \mu \mathrm{l}$ of each dilution was then placed into black 96 well plates for imaging (see "imaging parameters" section below).

\section{Dilution plating: infected Drosophila}

To determine the concentration of bacteria injected into individual flies, flies were suspended in $250 \mu$ l of $1 X$ phosphate buffered saline and homogenized (Krupp \& Levine, 2010). Homogenate 
was then used for stepwise serial dilutions. $5 \mu \mathrm{L}$ of each dilution in the series was then spot plated in triplicate on LB plates with $100 \mu \mathrm{g} / \mathrm{ml}$ ampicillin. Colonies were then counted for each dilution step to determine the concentration of CFUs per fly. For plasmid loss determinations, homogenate and serial dilution was prepared in the manner described above. Next $90 \mu$ of solution from each dilution in the series was plated on individual LB plates not supplemented ampicillin. Plates containing colonies were then counted for each dilution step to determine the concentration of CFUs per fly and imaged for luminescence output as described in the "imaging parameters" section. Thus, emissive and non-emissive colonies could be distinguished to determine the proportion of colonies that had lost the ilux plasmid.

\section{Imaging parameters}

All imaging analyses were performed in black 96-well plates (Grenier Bio One) prepared as described above, or on agar plates for plasmid loss studies. Biological replicates for different experiments were performed on different days. Plates containing flies were imaged immediately post-injection unless otherwise stated. Injected flies and agar plates containing ilux-Ecoli were imaged using an IVIS Lumina II (Xenogen) CCD camera chilled to $-90{ }^{\circ} \mathrm{C}$. The stage was kept at room temperature $\left(25^{\circ} \mathrm{C}\right)$ during the imaging session, and the camera was controlled using Living Image Software. The exposure time was $1 \mathrm{~s}-10$ min depending on the brightness of the sample, and the data binning levels were set to medium. Radiance was integrated over regions of interest and quantified to total flux values using the Living Image software. Raw luminescent images were analyzed using FIJI (Schindelin 2012). For plasmid loss studies, CFUs on plates were quantified by hand, luminescent colonies were quantified by importing for analysis and counting in FIJI.

\section{Data analysis}

All luminescence data was imported into R 3.6.0 for analysis and visualization (R Core Team. 2019, Wickham H. 2016, Dowle M(2019), Wickham H. 2021, Wickham et al., 2019). Model2 regressions were performed using the package Imodel2 1.7-3 (Legendre 2018). Euclidean dissimilarity and hierarchical clustering were performed using package TSclust 1.3.1 (Montero \& Vilar, 2014).

\section{References}

Ayres, J. S., Freitag, N., \& Schneider, D. S. (2008). Identification of Drosophila Mutants Altering Defense of and Endurance to Listeria monocytogenes Infection. Genetics, 178(3), 1807-1815. doi:10.1534/genetics.107.083782

Ayres, J. S., \& Schneider, D. S. (2008). A Signaling Protease Required for Melanization in Drosophila Affects Resistance and Tolerance of Infections. PLOS Biology, 6(12), e305. doi:10.1371/journal.pbio.0060305

Brandes, C., Plautz, J. D., Stanewsky, R., Jamison, C. F., Straume, M., Wood, K. V., . . Hall, J. C. (1996). Novel Features of Drosophila period Transcription Revealed by Real-Time Luciferase Reporting. Neuron, 16(4), 687-692. doi:https://doi.org/10.1016/S0896-6273(00)80088-4

Buchon, N., Silverman, N., \& Cherry, S. (2014). Immunity in Drosophila melanogaster - from microbial recognition to whole-organism physiology. Nature Reviews Immunology, 14(12), 796 810. doi:10.1038/nri3763

Carruthers, J., Lythe, G., López-García, M., Gillard, J., Laws, T. R., Lukaszewski, R., \& MolinaParís, C. (2020). Stochastic dynamics of Francisella tularensis infection and replication. PLOS 
Computational Biology, 16(6), e1007752. doi:10.1371/journal.pcbi.1007752

Chambers, M. C., Jacobson, E., Khalil, S., \& Lazzaro, B. P. (2019). Consequences of chronic bacterial infection in Drosophila melanogaster. PLOS ONE, 14(10), e0224440.

doi:10.1371/journal.pone.0224440

Chambers Moria, C., Jacobson, E., Khalil, S., Lazzaro Brian, P., \& Bäumler, A. J. (2014). Thorax Injury Lowers Resistance to Infection in Drosophila melanogaster. Infection and Immunity, 82(10), 4380-4389. doi:10.1128/IAI.02415-14

Contag, C. H., \& Bachmann, M. H. (2002). Advances in In Vivo Bioluminescence Imaging of Gene Expression. Annual Review of Biomedical Engineering, 4(1), 235-260.

doi:10.1146/annurev.bioeng.4.111901.093336

Cronin, M., Akin, A. R., Collins, S. A., Meganck, J., Kim, J.-B., Baban, C. K., . . Tangney, M. (2012). High Resolution In Vivo Bioluminescent Imaging for the Study of Bacterial Tumour Targeting. PLOS ONE, 7(1), e30940. doi:10.1371/journal.pone.0030940

Dowle, M., \& Srinivasan, A. (2019). data.table: Extension of 'data.frame'. R package version 1.12.2. https://CRAN.R-project.org/package=data.table

Duneau, D., Ferdy, J.-B., Revah, J., Kondolf, H., Ortiz, G. A., Lazzaro, B. P., \& Buchon, N. (2017). Stochastic variation in the initial phase of bacterial infection predicts the probability of survival in D. melanogaster. eLife, 6, e28298. doi:10.7554/eLife.28298

Ellner, S. P., Buchon, N., Dörr, T., \& Lazzaro, B. P. (2021). Host-pathogen immune feedbacks can explain widely divergent outcomes from similar infections. Proceedings of the Royal Society B: Biological Sciences, 288(1951), 20210786. doi:10.1098/rspb.2021.0786

Frochaux, M. V., Bou Sleiman, M., Gardeux, V., Dainese, R., Hollis, B., Litovchenko, M., .. . Deplancke, B. (2020). cis-regulatory variation modulates susceptibility to enteric infection in the Drosophila genetic reference panel. Genome Biology, 21(1), 6. doi:10.1186/s13059-019-1912-z

Gregor, C., Gwosch, K. C., Sahl, S. J., \& Hell, S. W. (2018). Strongly enhanced bacterial bioluminescence with the ilux operon for single-cell imaging. Proceedings of the National Academy of Sciences, 115(5), 962. doi:10.1073/pnas.1715946115

Hotson, A. G., \& Schneider, D. S. (2015). Drosophila melanogaster Natural Variation Affects Growth Dynamics of Infecting Listeria monocytogenes. G3 (Bethesda, Md.), 5(12), 2593-2600. doi:10.1534/g3.115.022558

Hunter, P. A., Dawson, S., French, G. L., Goossens, H., Hawkey, P. M., Kuijper, E. J., . . Piddock, L. J. V. (2010). Antimicrobial-resistant pathogens in animals and man: prescribing, practices and policies. Journal of Antimicrobial Chemotherapy, 65(suppl_1), i3-i17. doi:10.1093/jac/dkp433

Ja, W. W., Carvalho, G. B., Mak, E. M., de la Rosa, N. N., Fang, A. Y., Liong, J. C., . . Benzer, S. (2007). Prandiology of Drosophila and the CAFE assay. Proceedings of the National Academy of Sciences, 104(20), 8253. doi:10.1073/pnas.0702726104 James, M. L., \& Gambhir, S. S. (2012). A Molecular Imaging Primer: Modalities, Imaging 
Agents, and Applications. Physiological Reviews, 92(2), 897-965.

doi:10.1152/physrev.00049.2010

Johnson, M. B., \& Butterworth, F. M. (1985). Maturation and aging of adult fat body and oenocytesin Drosophila as revealed by light microscopic morphometry. Journal of Morphology, 184(1), 51-59. doi:https://doi.org/10.1002/jmor.1051840106

Kaskova, Z. M., Tsarkova, A. S., \& Yampolsky, I. V. (2016). 1001 lights: luciferins, luciferases, their mechanisms of action and applications in chemical analysis, biology and medicine.

Chemical Society Reviews, 45(21), 6048-6077. doi:10.1039/C6CS00296J

Krupp, J. J., \& Levine, J. D. (2010). Dissection of oenocytes from adult Drosophila melanogaster. Journal of visualized experiments : JoVE(41), 2242. doi:10.3791/2242

Kutzer, M. A. M., \& Armitage, S. A. O. (2016). The effect of diet and time after bacterial infection on fecundity, resistance, and tolerance in Drosophila melanogaster. Ecology and Evolution, 6(13), 4229-4242. doi:https://doi.org/10.1002/ece3.2185

Lack, J. B., Lange, J. D., Tang, A. D., Corbett-Detig, R. B., \& Pool, J. E. (2016). A Thousand Fly Genomes: An Expanded Drosophila Genome Nexus. Molecular Biology and Evolution, 33(12), 3308-3313. doi:10.1093/molbev/msw195

Lazzaro, B. P., Sackton, T. B., \& Clark, A. G. (2006). Genetic Variation in Drosophila melanogaster Resistance to Infection: A Comparison Across Bacteria. Genetics, 174(3), 1539-1554. doi:10.1534/genetics.105.054593

Legendre, P. (2018). Imodel2: Model II Regression. R package version 1.7-3. https://CRAN.R-project.org/package=Imodel2

Lemaitre, B., \& Hoffmann, J. (2007). The Host Defense of Drosophila melanogaster. Annual Review of Immunology, 25(1), 697-743. doi:10.1146/annurev.immunol.25.022106.141615

Love, A. C., \& Prescher, J. A. (2020). Seeing (and Using) the Light: Recent Developments in Bioluminescence Technology. Cell Chemical Biology, 27(8), 904-920.

doi:https://doi.org/10.1016/j.chembiol.2020.07.022

Massey, S., Johnston, K., Mott, T., Judy, B., Kvitko, B., Schweizer, H., . . Torres, A. (2011). In vivo Bioluminescence Imaging of Burkholderia mallei Respiratory Infection and Treatment in the Mouse Model. Frontiers in microbiology, 2, 174.

Montero, P., \& Vilar, J. A. (2014). TSclust: An R Package for Time Series Clustering. Journal of Statistical Software; Vol 1, Issue 1 (2015). doi:10.18637/jss.v062.i01

Morrissey, R., Hill, C., \& Begley, M. (2013). Shining light on food microbiology; applications of Lux-tagged microorganisms in the food industry. Trends in Food Science \& Technology, 32(1), 415. doi:https://doi.org/10.1016/j.tifs.2013.05.001

Moy, R. H., \& Cherry, S. (2013). Antimicrobial Autophagy: A Conserved Innate Immune Response in Drosophila. Journal of Innate Immunity, 5(5), 444-455. doi:10.1159/000350326

Pham, L. N., Dionne, M. S., Shirasu-Hiza, M., \& Schneider, D. S. (2007). A Specific Primed 
Immune Response in Drosophila Is Dependent on Phagocytes. PLOS Pathogens, 3(3), e26. doi:10.1371/journal.ppat.0030026

R Core Team. (2016). R: A Language and Environment for Statistical Computing. Vienna, Austria. Retrieved from https://www.R-project.org/

Sackton, T. B., Lazzaro, B. P., \& Clark, A. G. (2010). Genotype and Gene Expression Associations with Immune Function in Drosophila. PLOS Genetics, 6(1), e1000797. doi:10.1371/journal.pgen.1000797

Schindelin, J., Arganda-Carreras, I., Frise, E., Kaynig, V., Longair, M., Pietzsch, T., ... Cardona, A. (2012). Fiji: an open-source platform for biological-image analysis. Nature Methods, 9(7), 676-682. doi:10.1038/nmeth.2019

Schneider, D. (2000). Using Drosophila as a model insect. Nature Reviews Genetics, 1(3), 218 226. doi: $10.1038 / 35042080$

Schneider, D. S., \& Ayres, J. S. (2008). Two ways to survive infection: what resistance and tolerance can teach us about treating infectious diseases. Nature Reviews Immunology, 8(11), 889-895. doi:10.1038/nri2432

Stanewsky, R., Jamison, C. F., Plautz, J. D., Kay, S. A., \& Hall, J. C. (1997). Multiple circadian regulated elements contribute to cycling period gene expression in Drosophila. The EMBO Journal, 16(16), 5006-5018. doi:https://doi.org/10.1093/emboj/16.16.5006

Stempfl, T., Vogel, M., Szabo, G., Wülbeck, C., Liu, J., Hall, J. C., \& Stanewsky, R. (2002). Identification of Circadian-Clock-Regulated Enhancers and Genes of Drosophila melanogaster by Transposon Mobilization and Luciferase Reporting of Cyclical Gene Expression. Genetics, 160(2), 571-593. doi:10.1093/genetics/160.2.571

Troha, K., Im, J. H., Revah, J., Lazzaro, B. P., \& Buchon, N. (2018). Comparative transcriptomics reveals CrebA as a novel regulator of infection tolerance in $D$. melanogaster. PLOS Pathogens, 14(2), e1006847. doi:10.1371/journal.ppat.1006847

Wickham, H. (2016). Ggplot2: Elegant graphics for data analysis (2nd ed.) [PDF]. Springer International Publishing.

Wickham, H., Averick, M., Bryan, J., Chang, W., McGowan, L. D., François, R., . . Yutani H (2019). "Welcome to the tidyverse." Journal of Open Source Software, 4(43), 1686. doi: 10.21105/joss.01686.

Wickham, H., François, R., Henry, L., \& Müller, K (2021). dplyr A Grammar of Data Manipulation. R package version 1.0.6. https://CRAN.R-project.org/package=dplyr

Zambito, G., Chawda, C., \& Mezzanotte, L. (2021). Emerging tools for bioluminescence imaging. Current Opinion in Chemical Biology, 63, 86-94.

doi:https://doi.org/10.1016/j.cbpa.2021.02.005 